\title{
Resilience and occupational health of health care workers: a moderator analysis of organizational resilience and sociodemographic attributes
}

\author{
Lila Gonçalves ${ }^{1} \mathbb{D} \cdot$ Roser Sala ${ }^{1} \cdot$ José-Blas Navarro ${ }^{2}$
}

Received: 4 January 2021 / Accepted: 13 April 2021 / Published online: 2 June 2021

(c) The Author(s), under exclusive licence to Springer-Verlag GmbH Germany, part of Springer Nature 2021

\begin{abstract}
Objectives Although previous studies have proposed a positive influence of resilience on the mental and physical health of health care workers, empirical evidence on its relationship with occupational health remains scarce. This study aimed to analyze the relationship between individual resilience and several occupational health indicators, as well as exploring the moderating role of organizational resilience and sociodemographic attributes on this relationship.

Methods A cross-sectional design was used with a questionnaire applied to a sample of 325 workers from the Spanish health care sector.

Results Individual resilience was significantly associated with the indicators of occupational health. A direct effect of individual resilience on job satisfaction was found. The influence of resilience on the perception of fatigue and suffering from an illness was reverse. Age moderated the impact of resilience on the perception of stress and medical leave. Besides, organizational resilience proved to be an important adjustment variable in job satisfaction and perception of stress.

Conclusions The findings show the relevance to take both individual and organizational resilience into account when applying intervention programs to improve the occupational health of health care workers.
\end{abstract}

Keywords Resilience $\cdot$ Occupational health $\cdot$ Organizational resilience $\cdot$ Health care workers

\section{Introduction}

Resilience embodies the personal qualities that enable one to thrive in the face of adversity (Connor and Davidson 2003). Recently, resilience has become increasingly important in the workplace (Mills et al. 2013). According to Rook et al. (2018), resilience is a key characteristic of a successful employee in today's turbulent work environment.

In the healthcare sector, there are many stressful work circumstances. First, challenges can arise from complex

Lila Gonçalves

lila.goncalves@ciemat.es

1 CISOT Socio-Technical Research Centre of CIEMAT (Research Centre on Energy, Environment and Technologies), Mòdul de Recerca A, 1st Floor, Office MRA 123, Plaça del Coneixement, Universidad Autònoma de Barcelona, Bellaterra, 08193 Barcelona, Catalonia, Spain

2 Department of Psychobiology and Methodology of the Health Sciences, Universitat Autònoma de Barcelona, 08193 Bellaterra, Spain clinical cases or conflicts with patients. Health care professionals may also have to deal with organizational issues specific to their workplace. Finally, external organizational pressure such as scrutiny of practices is another source of stressful work (Robertson et al. 2016).

Frequent workplace stress can have a negative impact on the physical and mental wellbeing of health care professionals (Clark et al. 2016; McCann et al. 2013); while existing literature presents evidence that resilience has a positive impact on mental health. Aburn et al. (2016) identified good mental health as a proxy for resilience. Individuals with high levels of resilience tended to have less psychological health problems. Several studies link resilience with burnout, fatigue and stress in healthcare workers (Lebares et al. 2018; Mealer et al. 2017; Shatté et al. 2017; Winwood et al. 2013).

There are also studies that pointed out the positive impact of resilience on physical health (Ezeamama et al. 2016). Siu et al. (2009) found resilience was inversely related to physical symptoms and injuries at work among health care workers. 
Research has begun to explore the impact of resilience on employees as an organizational factor that produces important benefits, such as a reduction in absenteeism (Andolo 2013), and a better job satisfaction (Waddimba et al. 2016) in health care professionals. Nevertheless, the studies in this regard are scarce and it is necessary to go in-depth in other aspects such as the association between resilience and occupational health (occupational accidents, medical leaves).

\section{Sociodemographic variables}

Literature shows the importance of considering sociodemographic variables in the study of resilience. Age and gender are the most frequently connected to resilience (Lee et al. 2013a, b). Although some research reported a negative association between age and resilience (Beutel et al. 2009) most have shown a positive relationship (Chen et al. 2016; Gillespie et al. 2009; Martínez-Martí and Ruch 2017). Likewise, in some research, men were more resilient (CampbellSills et al. 2009), while in others, women were more resilient (Sull et al. 2015).

Research around resilience in health care organizations has focused on analyzing specific occupations, such as nurses and doctors. Sull et al. (2015) explored the association of work role and resilience and found that clinical staff reported lower levels of resilience than administrative staff.

Sociodemographic variables can also be considered important moderators of the relationship between resilience and health. Hu et al. (2015) found that age moderated the relationship between resilience and negative indicators of mental health. Gender was also a relevant moderator: female participants presented a stronger effect size on the relationship between resilience and all indicators of mental health.

No research has been found concerning the moderating effect of work role in the relationship between resilience and workers 'health.

\section{Organizational resilience}

Resilience in health care can be described as a characteristic of individuals, as well as a property of teams, and of the whole organization (Jeffcott et al. 2009). Recent health systems adversities such as the Ebola outbreak of 2014-2016 and the global financial crisis of 2008 have increased the interest in the concept of organizational resilience (Barasa et al. 2018). In addition, Hanefeld et al. (2018) highlight the need to make nowadays health systems more resilient.

Organizational resilience (OrRe) is organization's ability to survive and even strengthen itself in times of crisis. It is more visible after a natural disaster; however, in everyday life, organizations have to handle a variety of adversities in which organizational resilience is extremely important (Stephenson et al. 2010).
Organizational resilience assessment includes two factors: planning and adaptive capacity (Gonçalves et al. 2019). Planning implies the use of predetermined planning capacities for the continuity of the business and risk management initiatives. Adaptive capacity relates to the ability to deal with the organization's needs before they become critical, and it emerges as a result of strong leadership and culture (Lee et al. 2013a, b).

There are very few studies relating organizational resilience and individual resilience (IR). Resilient employees, when facilitated and supported by the organization, have the capability to apply resources to continually adapt and flourish at work, even when faced with challenging circumstances (Kuntz et al. 2016). As highlighted by Seville (2018) having good people and getting the best out of them, especially in times of great stress, is essential for organizational resilience.

In that sense, organizations can promote resilience by designing a work environment that supports people and positively influences the acquisition and use of resilience skills (Wachs et al. 2015), or enhancing employees' capacity to manage uncertainty (Weick and Sutcliffe 2015).

The health of the workforce is essential for a health system to respond to crisis, but in many cases, frontline health care workers themselves are amongst the most vulnerable individuals (Gostin and Friedman 2015). Worksite programs designed to improve resilience in health care employees have shown efficacy in improving resilience, quality of life and health behaviors (Werneburg et al. 2018). However, focusing all efforts on enhancing individual resilience may inadvertently lead organizations to reduce their focus on changing the environment to reduce exposure to adversity. Research that describes both personal and environmental factors that contribute to resilience is needed (Britt et al. 2016).

Despite the studies referred above, no specific findings have been identified regarding the influence of organizational resilience in the relationship between individual resilience and occupational health. This is a relevant research area, because in case of organizational crisis the health of the workers could be more impacted. According to Shoss et al. (2018), resilience is most important when job insecurity is high.

Based on the previous review, this study aims to analyze the relationship between individual resilience and several occupational health indicators of employees in the health care sector. It is also aimed at exploring the moderating role of sociodemographic variables and organizational resilience in this relationship. It was hypothesized that (1) individual resilience would have a direct impact on job satisfaction and inversely on the perception of stress, perception of fatigue, medical leave, accidents and illness, (2) the effect of individual resilience on occupational health would have a greater impact on women, (3) the effect of individual resilience 
on occupational health would have a greater impact in the elderly, (4) the work role would have a moderating role in the impact of individual resilience on occupational health, (5) organizational resilience would have a moderating effect on the influence of individual resilience on occupational health.

\section{Materials and methods}

\section{Participants}

This study presents an observational, non-randomized and cross-sectional design. To access the sample, the research team contacted the Catalan Association for Health Prevention, from where the study was disclosed to various healthcare organizations. Three hospitals accepted to participate. The entire staff of the participating healthcare organizations was invited to participate in the study. The employees were invited through various e-mails from the Human Resources Department, which indicated the interest of a wide participation. The final sample thus corresponds to a non-probabilistic convenience sample.
The sample consisted of 325 workers from three healthcare organizations in Barcelona, Spain (Table 1). The age of participants ranged from 20 to 67 years, with a mean of 44.5 years $(\mathrm{SD}=9.4)$, and $78.8 \%$ of them were women. From the total sample, $72.2 \%$ were professionals taking direct care of patients (doctors, nurses, ancillaries), while $27.8 \%$ were the administrative staff. Most of the participants (86.5\%) were full-time workers. The mean score of the seniority was 15.7 years in the organization $(\mathrm{SD}=9.0)$.

\section{Procedure}

This research was reviewed and approved by the Ethics Committee and the Occupational Health and Human Resources Committee. Anonymity and confidentiality of the data were guaranteed, in strict compliance with ethical research guidelines. The questionnaires were administered in paper or electronically. In one of the organizations, it was administered mainly in paper with the support of Human Resources and Occupational Health departments. A person in charge of both departments distributed the questionnaires in a sealed envelope to all the organization's personnel. The participants voluntarily deposited the questionnaires in a
Table 1 Description of the sample

\begin{tabular}{|c|c|}
\hline Variable & Total sample $(N=325)$ \\
\hline \multicolumn{2}{|l|}{ Gender-N $-N)$} \\
\hline Males & $69(21.2 \%)$ \\
\hline Females & $256(78.8 \%)$ \\
\hline $\mathrm{Age}-M(\mathrm{SD})$ & $44.52(9.38)$ \\
\hline Seniority $-M(\mathrm{SD})$ & $15.66(8.99)$ \\
\hline \multicolumn{2}{|l|}{ Work role $-N(\%)$} \\
\hline Patient care professionals & $226(72.17 \%)$ \\
\hline Administrative staff & $87(27.83 \%)$ \\
\hline \multicolumn{2}{|l|}{ Hours workday-N $-N)$} \\
\hline Full time ( $40 \mathrm{~h}$ per week) & $281(86.5 \%)$ \\
\hline Part time ( $20 \mathrm{~h}$ or less) & $18(5.5 \%)$ \\
\hline Others (more than $20 \mathrm{~h}$ ) & $26(8.0 \%)$ \\
\hline Planning $-M(\mathrm{SD})$ & $5.34(1.14)$ \\
\hline Adaptive capacity $-M(\mathrm{SD})$ & $4.36(1.40)$ \\
\hline Individual Resilience [0-4] & $3.08(0.51)$ \\
\hline \multicolumn{2}{|l|}{ Occupational Health [0-4] } \\
\hline Job satisfaction- $M(\mathrm{SD})$ & $2.96(0.81)$ \\
\hline Perception of stress- $M$ (SD) & $2.06(1.10)$ \\
\hline Perception of fatigue (Yes) $-N(\%)$ & $142(43.7 \%)$ \\
\hline Medical leave (Yes) $-N(\%)$ & $65(20.0 \%)$ \\
\hline Occupational accidents (Yes) $-N(\%)$ & $38(11.7 \%)$ \\
\hline Illness (Yes) $-N(\%)$ & $73(22.5 \%)$ \\
\hline
\end{tabular}

The minimum and maximum possible values are indicated between brackets 
mailbox provided for such purposes in the hospital. The entire data collection process took 3 weeks. A couple of reminders via e-mail were sent to encourage participation. In the other two organizations, the questionnaire was administered mainly electronically. For this, the Department of Human Resources sent a formal e-mail to the entire organization staff, providing all the relevant information of the study and inviting to access the questionnaire through a link. To encourage participation, a reminder email was sent before the end of the 3-week period. Those who did not have regular access to the computer at their usual workplace used the paper version, which was provided by the Occupational Health Department. Therefore, due to differences in the start-up time of the different organizations involved, data collection began in January 2018 and ended in April 2018.

\section{Instruments}

Individual resilience was evaluated with the short version of the CD-RISC-10 (Connor and Davidson 2003) adapted to Spanish by Soler et al. (2016). A 5-point Likert-type response format was used $(1=$ not true at all; $5=$ true nearly all of the time). Examples of the type of items contained in this scale are "I am able to adapt to change" and "I tend to recover after adversity". The alpha coefficient in the original scale was 0.87 . The same result was obtained in our sample.

Organizational resilience (OrRe) was measured using the Benchmark Resilience Tool short-form (BRT-13B) developed by Whitman et al. (2013) and validated in Spanish by Gonçalves et al. (2019). The BRT-13B has two factors: planning and adaptive capacity. This shorter questionnaire consists in 13 items on a Likert response scale with eight options from 1 (strongly disagree) to 8 (strongly agree). It includes questions such as "In our organizations we are mindful of how a crisis could affect us", "Our organization maintains sufficient resources to absorb unexpected change" or "Staff have the information and knowledge they need to respond to unexpected problems". Results of Cronbach's alpha obtained in the original scale validated in Spain was 0.81 in the planning factor and 0.92 in the adaptive capacity factor (Gonçalves et al. 2019). In our sample, Cronbach's alpha coefficient of 0.71 was obtained in planning, and 0.90 in adaptive capacity.

Occupational health questionnaire by Martín et al. (2007) was used to measure worker's health status based on the following attributes: job satisfaction, perception of stress, perception of fatigue, medical leave, occupational accident, illness. Each of these variables was measured with a single item: "I am very satisfied with my work", "I am very stressed", "Lately you have been more fatigued or tired than usual", "Have you had any medical leave in the last 12 months?", "Have you suffered a work-related accident in the last 5 years?", "Do you have a disease that has been diagnosed by a doctor or psychologist?". Job satisfaction and perception of stress were measured using a 5-point Likert scale $(0=$ totally disagree; $4=$ totally agree $)$. A dichotomous scale (no/yes) was used for the last four items. As these are unique items for each indicator, no internal consistency results are reported.

To minimize biases associated with the mono-method (such as social desirability bias), different actions were carried out as recommended by Podsakoff et al. (2003). First of all, all the information regarding the study and ethical standards were expressed on the first page of the questionnaire. Second, staff were allowed to fill out the questionnaire anonymously (in privacy), which may reduce bias and fear of reprisals by providing higher confidentiality. Third, data collection was done with reliable and well-established measures. Similarly, the use of mixed-mode formats allowed health professionals a greater flexibility to fit the survey within their busy schedules. For the same reason, instruments were used in their short-form versions.

\section{Data analysis}

Data analysis was carried out using Stata 14 using a sample of 325 valid cases. Because the main objective of the study was to estimate effects using multiple regression models, the minimum sample size was calculated following the proposal of Green (1991), assuming a medium effect size $\left(f^{2}=0.15\right)$ and a model with a maximum of 8 predictors (IR, interactions of IR with the two OrRe and a maximum of 5 adjusting terms). The result indicated that the sample should contain at least 108 participants. No restrictions were imposed to achieve a highest sample size as it finally was. The missing information was scarce, with a maximum of a $3.7 \%$ for predictors variables (work role) and a $0.6 \%$ for outcome measures (occupational accident). Cases with missing data were removed from analysis using listwise approach. Descriptive statistics and Pearson correlations were calculated to provide background information on the sample characteristics. To analyze the influence of IR on occupational health several regression models were estimated. Linear regression was applied to analyze job satisfaction and perception of stress, while logistic regression was employed in the analysis of the four binary outcomes: perception of fatigue, medical leave, occupational accident and illness.

The moderating or adjusting role of the two OrRe subscales, age, gender and work role was assessed through the next modelling sequence. First, it was analyzed if planning and adaptive OrRe moderated the influence of IR on each occupational health indicator. In case of non-significant results, the two interaction terms were eliminated from the model and it was evaluated if they were necessary as adjustment variables. To do this, the adjusted effect of IR was compared with the unadjusted one. If the change was greater 
than $10 \%$, the adjusted model was selected. Secondly, the process was repeated for the three sociodemographic variables (age, gender and work role) but, in this case, the analysis of the interaction between the moderator and individual resilience was carried out step by step, eliminating nonsignificant interactions one by one.

In the presence of interaction between IR and age, following Demaris (2004), the effect of IR was calculated for three representative values of age, one close to the minimum ( 25 years), one close to the maximum (65 years), and one close to the mean (45 years).

As odds ratios provided by logistic regression models are difficult to interpret, for the four binary outcomes an indirect estimation of the relative risk was obtained through Poisson regression models with robust estimation of the variance (Lin and Wei 1989).

To evaluate the internal consistency Cronbach's alpha coefficients were calculated.

\section{Results}

The sociodemographic characteristics of the sample are shown in Table 1.

Mean scores in Table 1 showed a perception of adequate OrRe, with greater results in the ability to plan crisis situations than the adaptive capacity of the organization. Health care workers obtained medium to high scores of IR. Health indicators results indicated that job satisfaction was high, and the perception of stress was medium. The perception of fatigue obtained the highest percentage and occupational accident had the lowest. The original scales used in the study are taken as a reference for these results.

The correlations obtained between resilience (individual and organizational) and occupational health variables are shown in Table 2 . IR was positively related with job satisfaction and with OrRe, while negative correlations were obtained with stress, fatigue and illness.
Table 3 shows the results of linear (Job satisfaction and Perception of stress) and logistic regression (Perception of fatigue, Medical leave, Occupational accidents and Illness). IR was significantly associated with the indicators of occupational health. A direct effect of IR on job satisfaction $(B=0.479 ; p<0.001)$ was found. The influence of IR on the perception of fatigue and suffering from an illness was reverse. Thus, a greater IR implies less fatigue $(\mathrm{OR}=0.590 ; p=0.027)$ and less presence of illness in health workers (OR $=0.494 ; p=0.011)$. Interaction effects between IR and age were found when the outcomes were stress $(p=0.031)$ and having had some medical leave $(p=0.029)$. The effect of IR on both outcomes was inverse and statistically significant only in older workers $(B=-0.817 ; p=0.007$ for the perception of stress) $(\mathrm{OR}=0.207 ; p=0.026$ for having had some medical leave). Figures 1 and 2 show graphically the moderation effect of age on the association between IR and stress and in the risk of a medical leave.

Regarding OrRe, results showed no moderation in the relationship between IR and occupational health. However, adjusting was necessary by planning and adaptive capacity of the organization in the linear models assessing the effect of IR both on job satisfaction and perception of stress. Age, gender and work role were also necessary as adjustment variables in the analysis of the influence of IR on the indicators of occupational health considered in this study.

The analysis of the indirect estimation of the relative risk (RR) through Poisson models for the four binary occupational health indicators revealed that increases in IR were associated with a decrease in the risk of perceived fatigue $(\mathrm{RR}=0.751 ; p=0.026)$ and the risk of being ill $(\mathrm{RR}=0.595 ; p=0.008)$. The association of IR with a medical leave and with having occupational accidents did not reach statistical significance in this analysis (Table 4).

Table 2 Correlations between individual resilience, organizational resilience and occupational health

\begin{tabular}{|c|c|c|c|c|c|c|c|c|}
\hline Measure & 2 & 3 & 4 & 5 & 6 & 7 & 8 & 9 \\
\hline 1. Individual resilience & $0.230 * *$ & $0.250^{* *}$ & $0.358 * *$ & $-0.111 *$ & $-0.120^{*}$ & $-0.030 *$ & 0.124 & $-0.142 *$ \\
\hline 2. Planning (OrRe) & & $0.633 * *$ & $0.246 * *$ & -0.057 & -0.053 & 0.063 & 0.035 & -0.070 \\
\hline 3. Adaptive capacity (OrRe) & & & $0.327 * *$ & $-0.128 *$ & $-0.113^{*}$ & -0.012 & -0.023 & -0.025 \\
\hline 4. Job satisfaction & & & & $-0.217^{* *}$ & $-0.203 * *$ & -0.099 & -0.006 & $-0.129 *$ \\
\hline 5. Perception of stress & & & & & $0.438 * *$ & $0.141 *$ & 0.067 & $0.159 * *$ \\
\hline 6. Perception of fatigue & & & & & & $0.132^{*}$ & $0.124^{*}$ & $0.164 * *$ \\
\hline 7. Medical leave & & & & & & & $0.204 * *$ & $0.191 * *$ \\
\hline 8. Occupational accidents & & & & & & & & 0.009 \\
\hline 9. Illness & & & & & & & & \\
\hline
\end{tabular}

${ }^{*} p<0.05 ; * * p<0.01$ 
Table 3 Linear/logistic regression of occupational health indicators on individual resilience

\begin{tabular}{|c|c|c|c|c|c|c|}
\hline & $B$ & & $|t|$ & & $p$ & CI $95 \% B$ \\
\hline Job satisfaction $^{\mathrm{a}}$ & 0.479 & & 5.57 & & $<0.001$ & $0.310 ; 0.648$ \\
\hline \multicolumn{7}{|l|}{ Perception of stress ${ }^{\mathrm{b}}$} \\
\hline Age $=25$ & 0.351 & & 1.20 & & 0.233 & $-0.227 ; 0.930$ \\
\hline Age $=45$ & -0.233 & & 1.84 & & 0.068 & $-0.483 ; 0.017$ \\
\hline \multirow[t]{2}{*}{ Age $=65$} & -0.817 & & 2.70 & & 0.007 & $-1.412 ;-0.222$ \\
\hline & OR & $\chi^{2}$ & & $p$ & CI $95 \%$ OR & $\mathrm{H}-\mathrm{L}(p)$ \\
\hline Perception of fatigue $^{c}$ & 0.590 & 4.92 & & 0.027 & $0.371 ; 0.941$ & 0.236 \\
\hline Medical leave $\mathrm{d}^{\mathrm{d}}$ & & & & & & 0.214 \\
\hline Age $=25$ & 3.592 & 3.09 & & 0.079 & $0.863 ; 14.957$ & \\
\hline Age $=45$ & 0.862 & 0.25 & & 0.615 & $0.484 ; 1.537$ & \\
\hline Age $=65$ & 0.207 & 4.97 & & 0.026 & $0.52 ; 0.827$ & \\
\hline Occupational accidents ${ }^{\mathrm{c}}$ & 2.191 & 4.10 & & 0.053 & $1.025 ; 4.680$ & 0.885 \\
\hline Illness ${ }^{\mathrm{c}}$ & 0.494 & 6.50 & & 0.011 & $0.287 ; 0.849$ & 0.873 \\
\hline
\end{tabular}

H-L: Hosmer-Leme show goodness of fit statistic

${ }^{a}$ Adjusted by planning, adaptative capacity, age, gender and work role

${ }^{\mathrm{b}}$ Adjusted by planning, adaptative capacity, gender and work role

${ }^{\mathrm{c}}$ Adjusted by age, gender and work role

${ }^{\mathrm{d}}$ Adjusted by gender and work role

Fig. 1 The moderating effect of age on the relationship between individual resilience and perception of stress

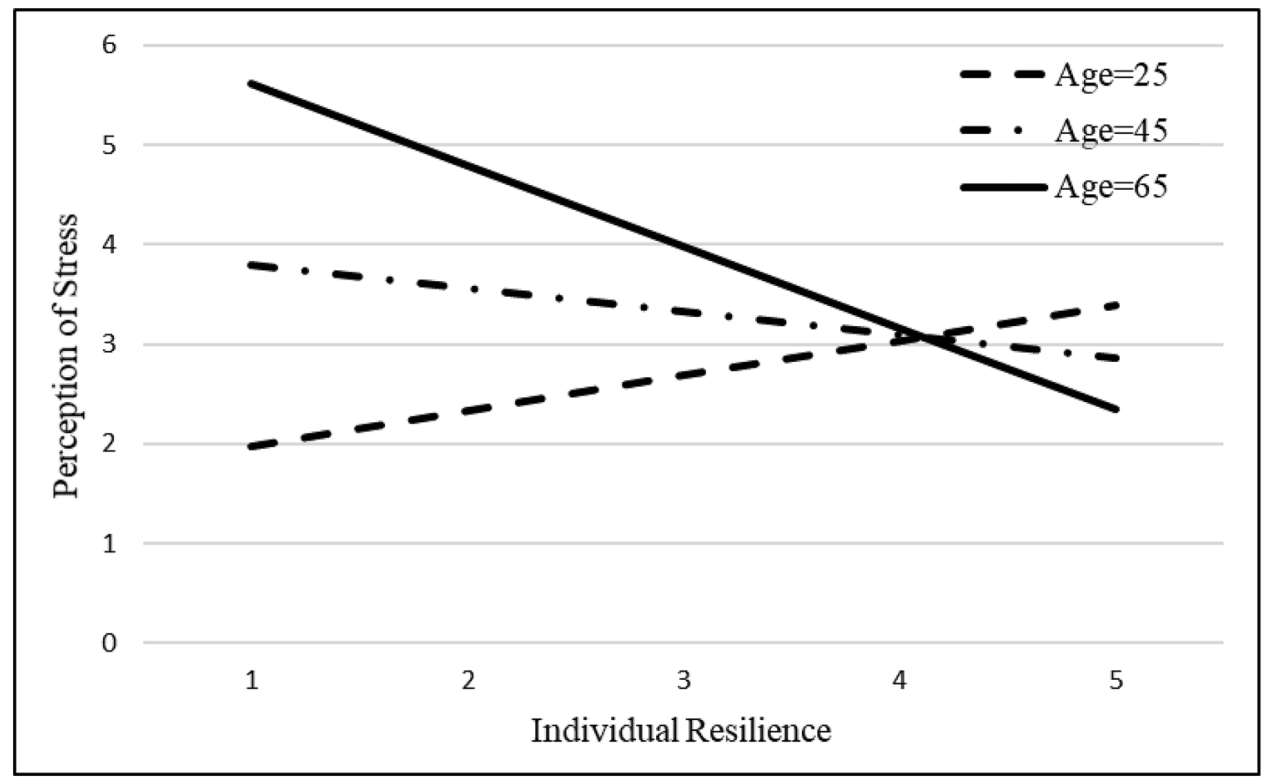

\section{Discussion}

This is the first research that explores the impact of IR on occupational health indicators, and the moderating role of OrRe and sociodemographic attributes. IR was significantly associated with occupational health. A direct effect of IR on job satisfaction was identified. The influence of IR on fatigue and the occurrence of an illness was inverse.
The effect on the two other occupational health indicators was moderated by age. Thus, the impact of IR on stress and medical leave was statistically significant for older employees. The hypotheses on gender and role moderation were not supported; however, both variables were found to play a relevant adjustment role in the relationship between IR and the following indicators: job satisfaction, perceived stress, fatigue and medical leave. Likewise, no moderation of OrRe on the relationship between IR and occupational 
Fig. 2 The moderating effect of age on the relationship between individual resilience and proportion of medical leave

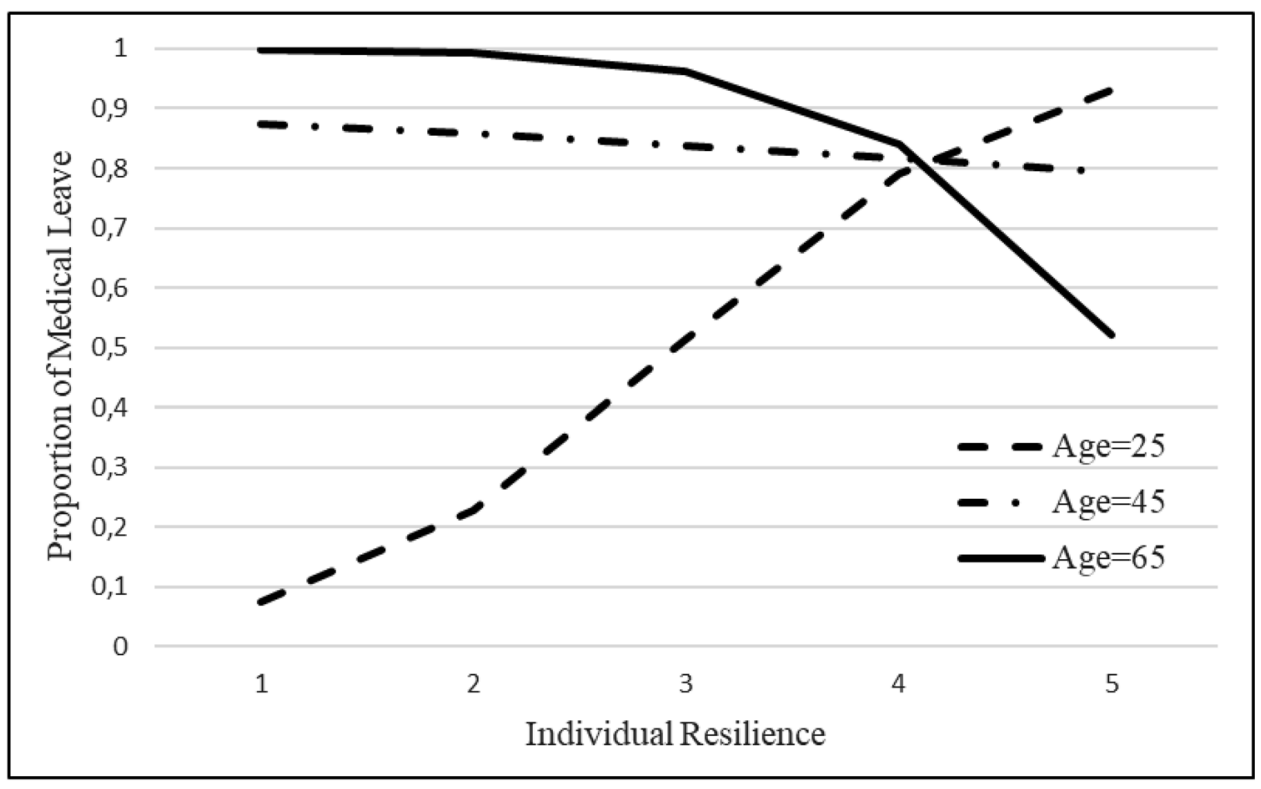

Table 4 Relative risk of binary occupational health indicators on individual resilience

\begin{tabular}{lllll}
\hline & $\mathrm{RR}$ & $\chi^{2}$ & $p$ & CI 95\% RR \\
\hline $\begin{array}{l}\text { Perception of fatigue }^{\mathrm{a}} \\
\text { Medical leave }^{\mathrm{b}}\end{array}$ & 0.751 & 4.97 & 0.026 & $0.584 ; 0.966$ \\
$\mathrm{Age}=25$ & & & & \\
$\mathrm{Age}=45$ & 2.654 & 2.16 & 0.142 & $0.722 ; 9.760$ \\
Age $=65$ & 0.907 & 0.15 & 0.701 & $0.550 ; 1.494$ \\
Occupational accidents $^{\mathrm{a}}$ & 1.998 & 3.53 & 0.060 & $0.971 ; 4.114$ \\
Illness $^{\mathrm{a}}$ & 0.595 & 7.11 & 0.008 & $0.406 ; 0.871$ \\
\hline
\end{tabular}

$R R$ relative risk

${ }^{\mathrm{a}}$ Adjusted by age, gender and work role

${ }^{\mathrm{b}}$ Adjusted by gender and work role

health was found. However, the two dimensions of OrRe were necessary as adjusting terms when assessing the effect of IR on job satisfaction and perception of stress.

Findings are consistent with other studies that have also identified high levels of resilience and high levels of job satisfaction in health care professionals (Zheng et al. 2017; Hudgins 2016; Kašpárková et al. 2018). Thus, individuals that are more resilient are more satisfied with their job.

As expected, a greater IR implies less fatigue and less presence of illness. A previous longitudinal study concluded that resilience reduced nurse fatigue (Saksvik-Lehouillier et al. 2012). Another study with health care workers pointed out that resilient individuals had a better perceived immune functioning and less physical/psychological symptoms (Lantman et al. 2017).

The impact of IR on stress and medical leave was moderated by age, being inverse and only significant in older workers. Similar results between IR and mental health indicators were obtained by $\mathrm{Hu}$ et al. (2015). Resilience has been associated with functional connectivity of brain regions involved in emotional flexibility, recovery capability, and inhibitory control process (Shi et al. 2019). According to Gloria and Steinhardt (2016) positive emotions may enhance resilience directly as well as indirectly through the mediating role of coping strategies. Some studies pointed out that older workers are better in terms of using adaptive emotion regulation strategies. Scheibe et al. (2016) support an older-age advantage in emotion regulation in health care professionals via enhanced use of adaptive strategies (e.g. positive reappraisal) and lower use of maladaptive strategies (e.g. rumination). Therefore, emotional regulation of resilient older workers could explain the results of this study.

Interestingly, IR was positively related to the two dimensions of OrRe. Workers with high resilience reported higher planning and adaptive capacity of their organizations. Although the results showed no moderation effect of OrRe, it was necessary to adjust the results for planning and adaptive capacity in job satisfaction and perception of stress. Matheson et al. (2016) propose that for a health care professional working in a challenging setting to be resilient, their personal resilience must align with workplace resilience. Anyway, our findings are in an exploratory phase and more research is required.

This study has several practical implications. First, an organizational resilience culture in hospitals should be developed, which means that Occupational Health and Safety Units give a high priority to resilience. The Human Resources department can play a significant role in creating a 'resilience culture' in which all people are aware of what resilience is, and in making interventions that can help to 
promote individual and organizational resilience (Branicki et al. 2016). Second, such interventions can be promoted through practical strategies and must be an integral part of an organization's policies (Salanova et al. 2016). Third, many of these techniques can help health care employees in changing their thoughts and emotions in order to be more positive, compassionate and aware (Pipe et al. 2012). Fourth, workplace programs designed to improve resilience can increase health behaviours (Werneburg et al. 2018) as well as health and well-being in health care workers (Robertson et al. 2016). Fifth, resilience should become a fundamental aspect for the creation of public health policies (Ziglio et al. 2017). Finally, improvements in resilience may foster a more dynamic workforce that is able to continue responding to long-lasting crises, such as the recent COVID-19 pandemic (Mahaffey et al. 2020).

The main limitation of the present study is that the analyses were performed only on the individual level. Meneghel et al. (2016) encourage researchers to expand the focus to the team and/or organizational level. The reduced number of participating organizations limits the generalization of the results. In fact, as noted by some authors, low survey response rates are a growing concern in research with health professionals (Cho et al. 2013). These authors suggest that researchers should make great effort to improve access to their target population by implementing appropriate incentive-based strategies and study design. As cross-sectional data have limitations regarding causality relationships, future longitudinal studies are needed, with different measures along time in the same hospital. Finally, the results were obtained from a single method of measurement with self-assessment questionnaires, therefore inferences about correlational and causal relationships can be inflated by the common method variance problem. According to Donaldson and Grant-Vallone (2002), it is important to emphasize that using multiple sources of data is a desirable strategy for avoiding the problem of being unable to rule out the problem of mono-method bias. Future studies will also benefit from the application of other data collection techniques such as interviews, focus groups and observations.

\section{Conclusion}

The main conclusion of the present study is that individual resilience is related to the occupational health of healthcare personnel, and that age moderates this relationship. Gender, job role and organizational resilience turned out to be important adjustment variables in the study of this relationship.

Supplementary Information The online version contains supplementary material available at https://doi.org/10.1007/s00420-021-01725-8.
Acknowledgements We are grateful to the Catalan Health Care Prevention Association for the support for access to hospitals.

Funding The Ministry of Economy and Competitiveness of Spain under predoctoral Grant (ID13-PREMM0-13) supported this work.

\section{Declarations}

Ethical approval This research project was reviewed by the Ethical Committee of the hospitals, which granted authorization to perform the study.

Conflict of interest No potential conflict of interest was reported by the authors.

\section{References}

Aburn G, Gott M, Hoare K (2016) What is resilience? An integrative review of the empirical literature. J Adv Nurs 72(5):980-1000. https://doi.org/10.1111/jan.12888

Andolo D (2013) From time to talk about workplace stress. In: Edmonstone J (ed) Personal resilience for healthcare staff, when the going gets tough. Radcliffe Publishing Ltd, London, pp 1-19

Barasa E, Mbau R, Gilson L (2018) What is resilience and how can it be nurtured? A systematic review of empirical literature on organizational resilience. Int J of Health Policy and Manag 7(6):491503. https://doi.org/10.15171/ijhpm.2018.06

Beutel ME, Glaesmer H, Decker O, Fischbeck S, Brähler E (2009) Life satisfaction, distress, and resiliency across the life span of women. Menopause J North Am Menopause Soc 16(6):1132-1138. https:// doi.org/10.1097/gme.0b013e3181a857f8

Branicki L, Steyer V, Sullivan-Taylor B (2016) Why resilience managers aren't resilient, and what human resource management can do about it. Int J of Hum Resour Manag 5192:1-26. https://doi.org/ 10.1080/09585192.2016.1244104

Britt TW, Shen W, Sinclair RR, Grossman MR, Klieger DM (2016) How much do we really know about employee resilience? Ind Organ Psychol 9(2):378-404. https://doi.org/10.1017/iop.2015. 107

Campbell-Sills L, Forde DR, Stein MB (2009) Demographic and childhood environmental predictors of resilience in a community sample. J Psychiat Res 43(12):1007-1012. https://doi.org/10.1016/j. jpsychires.2009.01.013

Chen X, Wang Y, Yan Y (2016) The essential resilience scale: instrument development and prediction of perceived health and behaviour. Stress Heal 32(5):533-542. https://doi.org/10.1002/smi.2659

Cho YI, Johnson TP, VanGeest JB (2013) Enhancing surveys of health care professionals a meta-analysis of techniques to improve response. Eval Health Prof 36(3):382-407

Clark MM, Jenkins SM, Hagen PT, Riley BA, Eriksen CA, Heath AL, Olsen KD (2016) High stress and negative health behaviors. J Occup Environ Med 58(9):868-873. https://doi.org/10.1097/jom. 0000000000000826

Connor KM, Davidson JRT (2003) Development of a new resilience scale: the Connor-Davidson resilience scale (CD-RISC). Depress Anxiety 18(2):76-82. https://doi.org/10.1002/da.10113

Demaris A (2004) Wiley series in probability and statistics. In: Regression with social data: modeling continuous and limited response variables. Wiley. https://doi.org/10.1002/0471677566

Donaldson SI, Grant-Vallone EJ (2002) Understanding self-report bias in organizational behavior research. J Business Psychol 17(2):245-260 
Ezeamama AE, Elkins J, Simpson C, Smith SL, Allegra JC, Miles TP (2016) Indicators of resilience and healthcare outcomes: findings from the 2010 health and retirement survey. Qual Life Res 25(4):1007-1015. https://doi.org/10.1007/s11136-015-1144-y

Gillespie BM, Chaboyer W, Wallis M (2009) The influence of personal characteristics on the resilience of operating room nurses: a predictor study. Int J Nurs Stud 46(7):968-976. https://doi.org/ 10.1016/j.ijnurstu.2007.08.006

Gloria CT, Steinhardt MA (2016) Relationships among positive emotions, coping, resilience and mental health. Stress Heal 32(2):145-156. https://doi.org/10.1002/smi.2589

Gonçalves L, Navarro JB, Sala R (2019) Spanish validation of the benchmark resilience tool (short-form version) to evaluate organisational resilience. Saf Sci 111:94-101. https://doi.org/ 10.1016/j.ssci.2018.09.015

Gostin LO, Friedman EA (2015) A retrospective and prospective analysis of the West African Ebola virus disease epidemic: robust national health systems at the foundation and an empowered WHO at the apex. Lancet 385(9980):1902-1909. https:// doi.org/10.1016/S0140-6736(15)60644-4

Green SB (1991) How many subjects does it take to do a regression analysis. Multivariate Behav Res 26(3):499-510. https://doi. org/10.1207/s15327906mbr2603_7 (PMID: 26776715)

Hanefeld J, Mayhew S, Legido-Quigley H, Martineau F, Karanikolos M, Blanchet K et al (2018) Towards an understanding of resilience: responding to health systems shocks. Health Policy Plan 33(10):1144-1144. https://doi.org/10.1093/heapol/czy087

Hu T, Zhang D, Wang J (2015) A meta-analysis of the trait resilience and mental health. Pers Individ Dif 76:18-27. https://doi.org/ 10.1016/j.paid.2014.11.039

Hudgins TA (2016) Resilience, job satisfaction and anticipated turnover in nurse leaders. J Nurs Manag 24(1):E62-E69. https://doi. org/10.1111/jonm.12289

Jeffcott SA, Ibrahim JE, Cameron PA (2009) Resilience in healthcare and clinical handover. Qual Saf Heal Care 18(4):256-260. https://doi.org/10.1136/qshc.2008.030163

Kašpárková L, Vaculík M, Procházka J, Schaufeli WB (2018) Why resilient workers perform better: the roles of job satisfaction and work engagement. J Workplace Behav Health 33(1):43-62. https://doi.org/10.1080/15555240.2018.1441719

Kuntz JR, Näswall K, Malinen S (2016) Resilient employees in resilient organizations: flourishing beyond adversity. Ind Organ Psychol Perspect Sci Pract 9(2):456-462. https://doi.org/10.1017/ iop.2016.39

Lantman M, Mackus M, Otten L, de Kruijff D, van de Loo A, Kraneveld A, Verster J (2017) Mental resilience, perceived immune functioning, and health. J Multidiscip Healthc 10:107-112. https://doi.org/10.2147/JMDH.S130432

Lebares CC, Guvva EV, Ascher NL, O'Sullivan PS, Harris HW, Epel ES (2018) Burnout and stress among US surgery residents: psychological distress and resilience. J Am Coll Surg 226(1):80-90. https://doi.org/10.1016/j.jamcollsurg.2017.10.010

Lee AV, Vargo J, Seville E (2013a) Developing a tool to measure and compare organizations' resilience. Nat Hazards Rev 14:29-34. https://doi.org/10.1061/(ASCE)NH.1527-6996.0000075

Lee JH, Nam SK, Kim AR, Kim B, Lee MY, Lee SM (2013b) Resilience: a meta-analytic approach. J Couns Dev 91(3):269-279. https://doi.org/10.1002/j.1556-6676.2013.00095.x

Lin DY, Wei LJ (1989) The robust inference for the Cox proportional hazards model. J Am Stat Assoc 84:1074-1078

Mahaffey BL, Mackin DM, Rosen J, Schwartz RM, Taioli E, Gonzalez A (2020) The disaster worker resiliency training program: a randomized clinical trial. Int Arch Occup Environ Health 1-13. https://doi.org/10.1007/s00420-020-01552-3

Martín J, Luceño L, Jaén M, Rubio S (2007) Relación entre factores psicosociales adversos, evaluados a través del cuestionario multidimensional Decore, y salud laboral deficiente. Psicothema 19(1):95-101

Martínez-Martí ML, Ruch W (2017) Character strengths predict resilience over and above positive affect, self-efficacy, optimism, social support, self-esteem, and life satisfaction. J Posit Psychol 12(2):110-119. https://doi.org/10.1080/17439760.2016.1163403

Matheson C, Robertson HD, Elliott AM, Iversen L, Murchie P (2016) Resilience of primary healthcare professionals working in challenging environments: a focus group study. $\mathrm{Br} \mathrm{J}$ Gen Pract 66(648):e507-e515. https://doi.org/10.3399/bjgp16X685285

McCann CM, Beddoe E, McCormick K, Huggard P, Kedge S, Adamson C, Huggard J (2013) Resilience in the health professions: a review of recent literature. Int J Wellbeing 3(1):60-81. https://doi. org/10.5502/ijw.v3i1.4

Mealer M, Jones J, Meek P (2017) Factors affecting resilience and development of posttraumatic stress disorder in critical care nurses. Am J Crit Care 26(3):184-192. https://doi.org/10.4037/ ajcc2017798

Meneghel I, Borgogni L, Miraglia M, Salanova M, Martínez IM (2016) From social context and resilience to performance through job satisfaction: a multilevel study over time. Hum Relations 69(11):2047-2067. https://doi.org/10.1177/0018726716631808

Mills MJ, Fleck CR, Kozikowski A (2013) Positive psychology at work: a conceptual review, state-of-practice assessment, and a look ahead. J Posit Psychol 8(2):153-164. https://doi.org/10.1080/ 17439760.2013.776622

Pipe TB, Buchda VL, Launder S, Hudak B, Hulvey L, Karns KE, Pendergast D (2012) Building personal and professional resources of resilience and agility in the healthcare workplace. Stress Heal 28(1):11-22. https://doi.org/10.1002/smi.1396

Podsakoff PM, MacKenzie SB, Lee JY, Podsakoff NP (2003) Common method biases in behavioral research: a critical review of the literature and recommended remedies. J Appl Psychol 88(5):879-903

Robertson HD, Elliott AM, Burton C, Iversen L, Murchie P, Porteous T, Matheson C (2016) Resilience of primary healthcare professionals: a systematic review. Br J Gen Pract 66(647):e423-e433. https://doi.org/10.3399/bjgp16X685261

Rook C, Smith L, Johnstone J, Rossato C, López Sánchez GF, Díaz Suárez A, Roberts J (2018) Reconceptualising workplace resilience-a cross-disciplinary perspective. An Psicol 34(2):332. https://doi.org/10.6018/analesps.34.2.299371

Saksvik-Lehouillier I, Bjorvatn B, Hetland H, Sandal GM, Moen BE, Magerøy N, Pallesen S (2012) Personality factors predicting changes in shift work tolerance: a longitudinal study among nurses working rotating shifts. Work Stress 26(2):143-160. https://doi. org/10.1080/02678373.2012.686344

Salanova M, Llorens S, Martínez IM (2016) Contributions from positive organizational psychology to develop healthy and resilient organizations. Papeles Del Psicólogo 37(3):177-184

Scheibe S, Spieler I, Kuba K (2016) An older-age advantage? Emotion regulation and emotional experience after a day of work. Work Aging Retire 2(3):307-320. https://doi.org/10.1093/workar/ waw010

Seville E (2018) Building resilience: how to have a positive impact at the organizational and individual employee level. Dev Learn Organ 32(3):15-18. https://doi.org/10.1108/DLO-09-2017-0076

Shatté A, Perlman A, Smith B, Lynch WD (2017) The positive effect of resilience on stress and business outcomes in difficult work environments. J Occup Environ Med 59(2):135-140. https://doi. org/10.1097/JOM.0000000000000914

Shi L, Sun J, Wei D, Qiu J (2019) Recover from the adversity: functional connectivity basis of psychological resilience. Neuropsychologia 122(61872301):20-27. https://doi.org/10.1016/j.neuro psychologia.2018.12.002

Shoss MK, Jiang L, Probst TM (2018) Bending without breaking: a two-study examination of employee resilience in the face of job 
insecurity. J Occup Health Psychol 23(1):112-126. https://doi. org/10.1037/ocp0000060

Siu OL, Hui CH, Phillips DR, Lin L, Wong T, Shi K (2009) A study of resiliency among Chinese health care workers: capacity to cope with workplace stress. J Res Pers 43(5):770-776. https://doi.org/ 10.1016/j.jrp.2009.06.008

Soler Sánchez MI, Meseguer de Pedro M, García Izquierdo M (2016) Propiedades psicométricas de la versión española de la escala de resiliencia de 10 ítems de Connor-Davidson (CD-RISC 10) en una muestra multiocupacional. Rev Latinoam Psicol 48(3):159-166. https://doi.org/10.1016/j.rlp.2015.09.002

Stephenson A, Vargo J, Seville E (2010) Measuring and comparing organisational resilience in Auckland. Aust J Emerg Manag 25(2):27-32

Sull A, Harland N, Moore A (2015) Resilience of health-care workers in the UK; a cross-sectional survey. J Occup Med Toxicol 10(1):1-8. https://doi.org/10.1186/s12995-015-0061-x

Wachs P, Saurin TA, Righi AW, Wears RL (2015) Resilience skills as emergent phenomena: a study of emergency departments in Brazil and the United States. Appl Ergon 56:227-237. https://doi.org/10. 1016/j.apergo.2016.02.012

Waddimba AC, Scribani M, Hasbrouck MA, Krupa N, Jenkins P, May JJ (2016) Resilience among employed physicians and mid-level practitioners in upstate New York. Health Serv Res 51(5):17061734. https://doi.org/10.1111/1475-6773.12499

Weick CE, Sutcliffe K (2015) Managing the unexpected: sustained performance in a complex world. Wiley, USA
Werneburg BL, Jenkins SM, Friend JL, Berkland BE, Clark MM, Rosedahl JK, Sood A (2018) Improving resiliency in healthcare employees. Am J Health Behav 42(1):39-50. https://doi.org/10. 5993/AJHB.42.1.4

Whitman ZR, Kachali H, Roger D, Vargo J, Seville E (2013) Short-form version of the Benchmark Resilience Tool (BRT-53). Meas Bus Excell 17(3):3-14. https://doi.org/10.1108/MBE-05-2012-0030

Winwood PC, Colon R, McEwen K (2013) A Practical measure of workplace resilience: developing the resilience at work scale. J Occup Environ Med 55(10):1205-1212. https://doi.org/10.1097/ JOM.0b013e3182a2a60a

Zheng Z, Gangaram P, Xie H, Chua S, Ong SBC, Koh SE (2017) Job satisfaction and resilience in psychiatric nurses: a study at the Institute of Mental Health, Singapore. Int J Ment Health Nurs 26(6):612-619. https://doi.org/10.1111/inm.12286

Ziglio E, Azzopardi-Muscat N, Briguglio L (2017) Editorials: resilience and 21 st century public health. Eur J Public Health 27(5):789-790. https://doi.org/10.1093/eurpub/ckx116

Publisher's Note Springer Nature remains neutral with regard to jurisdictional claims in published maps and institutional affiliations. 